\title{
The effect of therapeutic instrumental music performance method on upper extremity functions in adolescent cerebral palsy
}

\author{
Bilinc Dogruoz Karatekin ${ }^{1}$ (D) Afitap Icagasioglu²
}

Received: 4 November 2020 / Accepted: 29 January 2021 / Published online: 15 February 2021

(c) Belgian Neurological Society 2021

\begin{abstract}
The aim of the study is to investigate the improvement of upper extremity functions with piano training of adolescent cerebral palsy. Nine adolescent cerebral palsy patients admitted to the Pediatric Disability Clinic between 2018 and 2020 and 9 healthy adolescent volunteers as control group were included. Therapeutic Instrumental Music Performance method was applied 2 days a week, 3 months in 40-min sessions. Before/after intervention, MACS, Box Block Test, Nine-Hole Peg Test, Jamar hand dynamometer and key pressing force of fingers were evaluated with Cubase MIDI program. Five of our patients included in the study were spastic hemiplegic and 4 were spastic diplegic cerebral palsy. All measurements made after intervention were found to be statistically significant compared to the measurements made before piano training $(p<0.05)$. The fingers that improved the most in the key pressing force of the fingers were found as the right hand 4th, left hand the 4th and 5th fingers $(p<0.01)$. A significant strong negative relationship was detected between the Box Block Test and the Nine-Hole Peg Test $(p<0.001)$. With therapeutic instrumental music performance method, functional gains can be achieved in the grip strength, strengths of the fingers, gross and fine motor skills of adolescent cerebral palsy patients. Further studies are needed to establish a piano training protocol in neurological music therapy.
\end{abstract}

Keywords Cerebral palsy · Neurological music therapy · Piano training $\cdot$ Therapeutic instrumental music performance Upper extremity rehabilitation

\section{Introduction}

Cerebral palsy (CP) describes a group of permanent disorders of the development of movement and posture, causing activity limitation, that are attributed to non-progressive disturbances that occurred in the developing fetal or infant brain [1].

Hand is very important in daily life in patients with CP. Affected upper limb functions are one of the most important factors affecting daily life activities in patients with CP.

Bilinc Dogruoz Karatekin

bilincdogruoz@hotmail.com

Afitap Icagasioglu

afitapi@gmail.com

1 Department of Physical Medicine and Rehabilitation, Istanbul Medeniyet University Goztepe Prof Dr Suleyman Yalcin City Hospital, Istanbul, Turkey

2 Faculty of Medicine, Department of Physical Medicine and Rehabilitation, Istanbul Medeniyet University, Istanbul, Turkey
Reaching, grasping and releasing cannot be performed in a normal pattern due to limited range of motion and problems in isolated finger and thumb mobility [2].

Boinier et al. [3] stated that hand functions of children with hemiplegic cerebral palsy are very limited and they have difficulty in performing complex tasks in daily activities. Levitt et al. [4] also emphasized that hand functions are important in children with CP and that the affected upper limb functions are the most important factor affecting daily life activities.

During the rehabilitation process that starts from the moment of diagnosis and lasts for many years, patients usually discontinue their therapy programs and follow-ups during the adolescent period.

Numerous clinical studies have shown that auditory rhythm and music can be used effectively for specific therapeutic purposes [5-7]. Given the fact that it is not easy to implement the rehabilitation program for patients without a good concentration and motivation, the advantages of music therapy are more emphasized today. Music therapy increases the effectiveness of the treatment and maximizes its effects 
by stimulating the person's interest and increasing their participation in the treatment.

The concept of music therapy in rehabilitation has been documented by Thaut on a neuroscientific basis, standardized and a field known as neurological music therapy (NMT) has been defined [8]. Therapeutic instrumental music performance (TIMP) is one of three techniques addressing motor rehabilitation in neurological music therapy. TIMP uses musical instruments to help patients train impaired motor functions and regain functional movement patterns. Although the effect of NMT has been shown with various studies, the number of studies involving TIMP is insufficient $[9,10]$.

The aim of this study is to investigate the development of upper extremity motor functions of adolescent patients with cerebral palsy, to prevent the treatment interruptions as the age progresses by adding music to the rehabilitation process, to enable children with cerebral palsy to participate in daily life activities by increasing their hand functions and as a result, to increase their living standards.

Fig. 1 CONSORT flow diagram of the sampling stages

\section{Material and methods}

Among the 300 patients with cerebral palsy followed in the rehabilitation clinic of the university hospital, those between the ages of 11-18 were reached. Of the 52 adolescent cerebral palsy patients reached, 12 met the inclusion criteria. 3 patients dropped the rehabilitation sessions subsequently ( 2 of them were due to ongoing coronavirus epidemic and lockdown, 1 due to transfer difficulty), the study was completed with 9 patients.

9 adolescent patients diagnosed with cerebral palsy and 9 age and gender-matched controls who were admitted to University Hospital Pediatric Rehabilitation Clinic were included. Flow diagram of the study is shown at Fig. 1.

The study included patients between the ages of 11 and 18, diagnosed with CP, with upper limb involvement, not previously trained in music, having a cognitive level to understand and execute the commands (studying in a normal educational institution, middle school or high school appropriate for his age), and who agreed to participate the study. Four spastic diplegic cerebral palsy
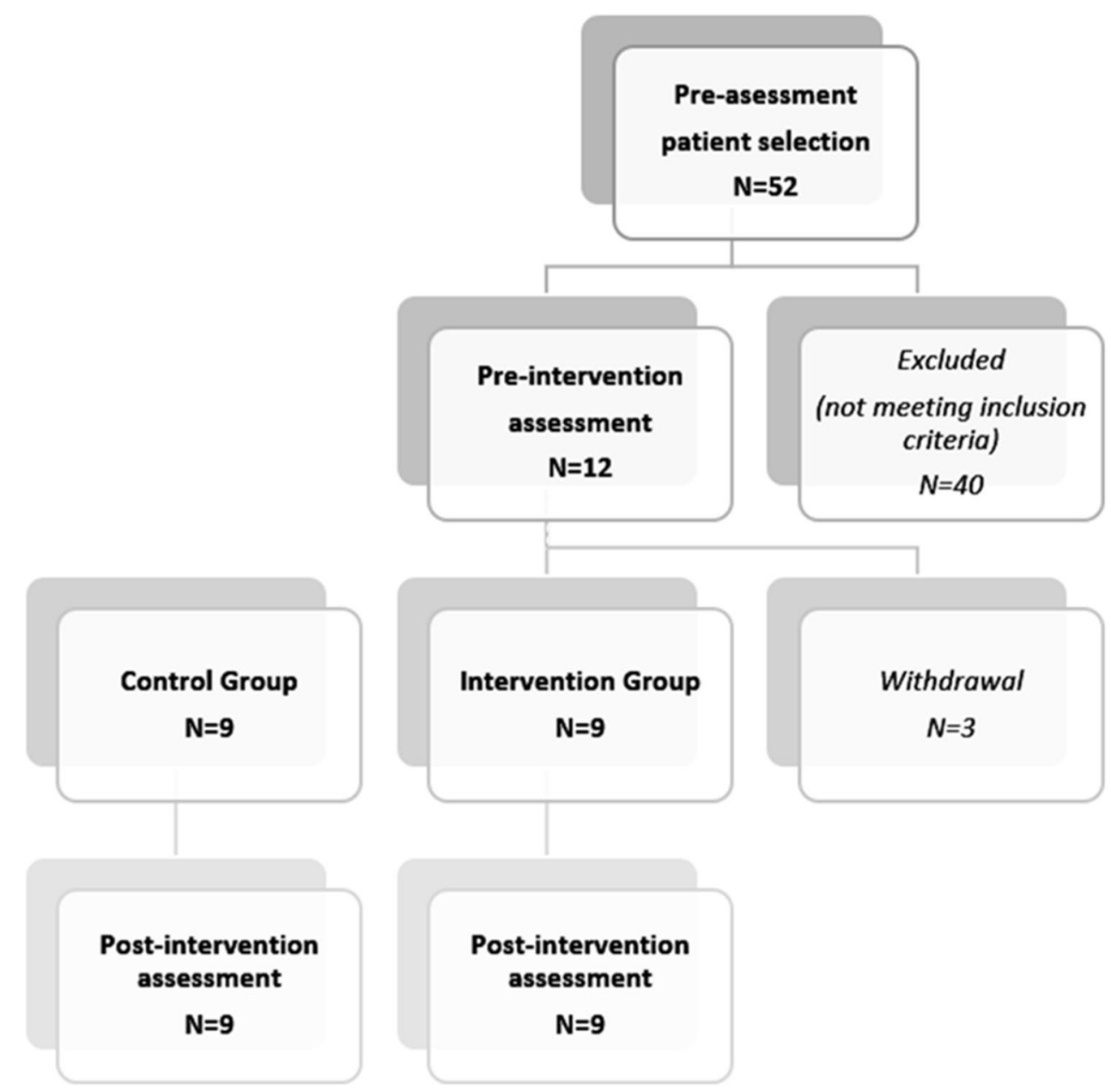
patients included in the study were patients with minor hand involvement.

Prior to the study, the approval of Medical Ethics Committee of our University Hospital was obtained (Decision no: 2018/0199). This study is registered to clinicaltrials. gov (ID: NCT03778398). Written informed consent for participation and publication from patients and caregivers has been obtained.

Patients were evaluated by the same researcher before and after the training. Detailed locomotor system examinations of the patients were recorded.

Hand functions of the patients in daily activities, before and after 3 months of training, evaluated with the Manual Ability Classification System (MACS), the gross motor functions of hand evaluated with the wooden box block test (BBT) and the upper extremity function and finger dexterity evaluated with the 9-hole peg test (NHPT), grip strength evaluated with the Jamar hand dynamometer, the key pressing forces of five individual fingers were evaluated with the Cubase MIDI program. BBT, NHPT, Jamar hand dynamometer and key pressing strength of fingers of nine healthy adolescents were also recorded.

Box block test, 9-hole peg test and grip strength measurement were repeated twice on the table at the height of the elbows, for the right and left hand, and the averages of the tests were taken.

\section{Evaluation tools}

\section{Manual Ability Classification System (MACS)}

The Manual Ability Classification System (MACS) [11] determines how patients with $\mathrm{CP}$ use their hands while holding objects in daily activities. MACS defines five levels. The determination of the levels is based on the child's ability to hold objects on his own and the need for help in performing hand-related activities in daily life. MACS is applied between the ages of 1-4 and 4-18. The validity and reliability of our country was done by Akpınar et al. [12].

\section{Box block test (BBT)}

Box block test is valid and reliable tool to evaluate the gross manual dexterity unilaterally [13]. It consists of a wooden box divided into two equal parts of $53.7 \mathrm{~cm} \times 25.4 \mathrm{~cm}$ and 150 blocks with $2.5 \mathrm{~cm}$ each side. The patient is asked to grasp the wooden blocks with his hand and pass it from one side of the box to the other; the number of wooden blocks transferred in $60 \mathrm{~s}$ is calculated.

\section{Nine-hole peg test (NHPT)}

It is a simple, fast, manual dexterity test with validity and reliability [14]. It is particularly sensitive to changes in upper limb performance. The test material consists of nine small bars made in standard size and a nine-hole board on which to place them. NHPT is applied in sitting position. The patient is asked to string the nine bars inside the box resting on the table into the holes of the other box as quickly as possible and remove them immediately after finishing. Time is measured with a stopwatch and is started when the hand touches the first bar and is finished when the last bar is placed in the box.

\section{Hand grip strength}

In the measurement of hand grip strength, Jamar hand dynamometer, which has high validity and reliability, and therefore accepted as the gold standard and whose norm values between the ages of 6 and 19 are available in the literature, have been used [15-17]. Jamar hydraulic hand dynamometer has the feature of showing hand grip strength with its analog indicator over two separate units. It can measure in pounds and kilograms. The measuring range is $200 \mathrm{lb}$ or $90 \mathrm{~kg}$.

\section{Cubase MIDI program}

Cubase is a Digital Audio Processing Station (DAW) software developed by the German company Steinberg and used for music recording and editing. Cubase LE 9.5 version was used to create, edit and analyze MIDI recordings (Fig. 2). The speed of key pressing can be measured in the Cubase program's MIDI interface. The keystroke speed data transferred from the digital piano to the program indicates the pressing power to the keys. Changes in values taken before and after the training reflect the effectiveness of the intervention. The measurement was made after 3 trials.

\section{Customized piano training program}

In this study, therapeutic instrumental music performance technique (TIMP), one of the three techniques of Thaut that can be used in motor rehabilitation in neurological music therapy (NMT), has been used.

Patients received a total of 3 months of customized piano training in 40-min sessions 2 days a week. The trainer is an advanced piano player and physiatrist and the piano used in the training is an electronic piano (Casio Celviano(C) with semi-weighted keys (Fig. 3).

Additionally, the keys are marked with different colored adhesive papers, so the notes are symbolized with colors to 


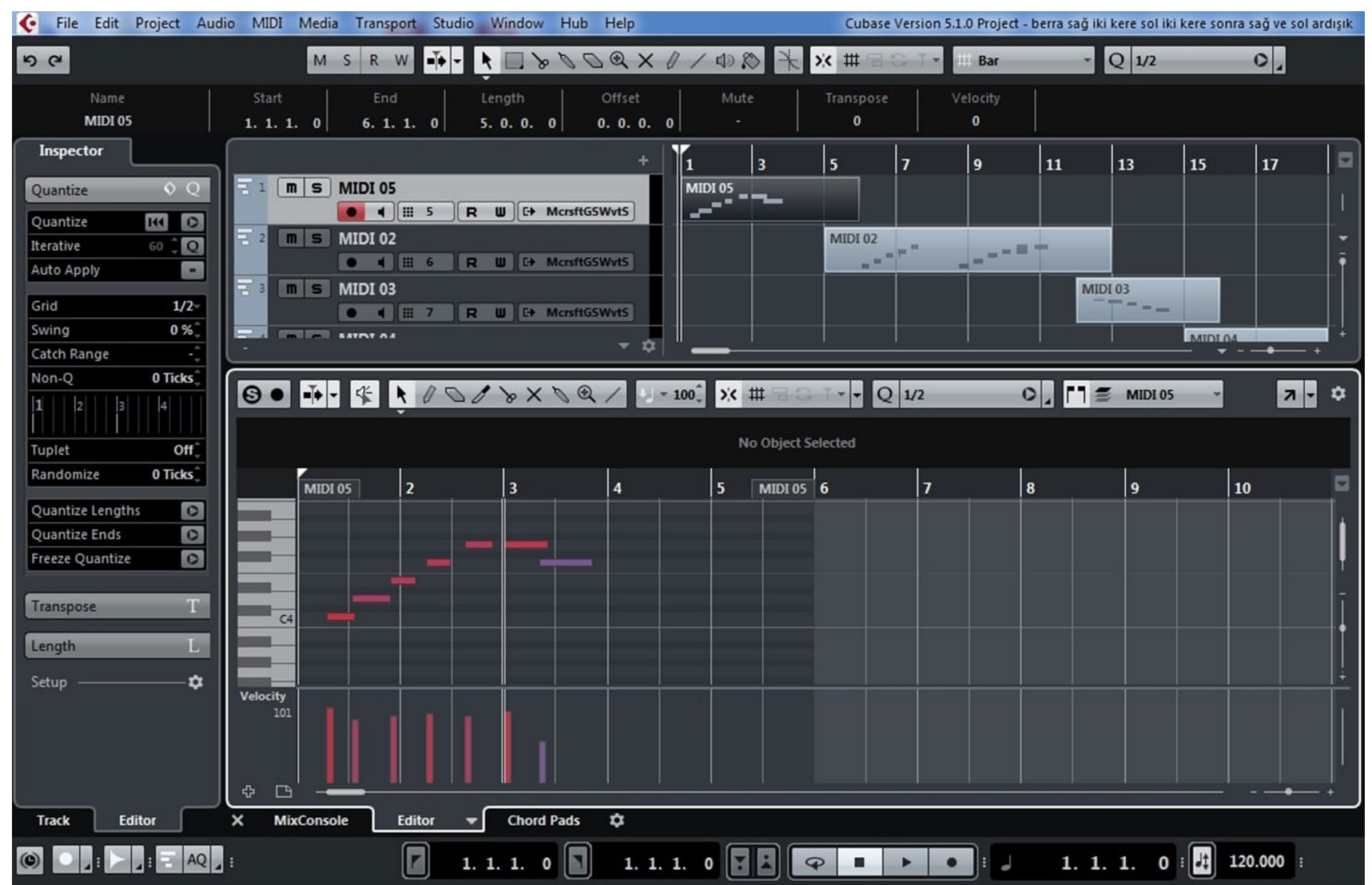

Fig. 2 Cubase MIDI program interface

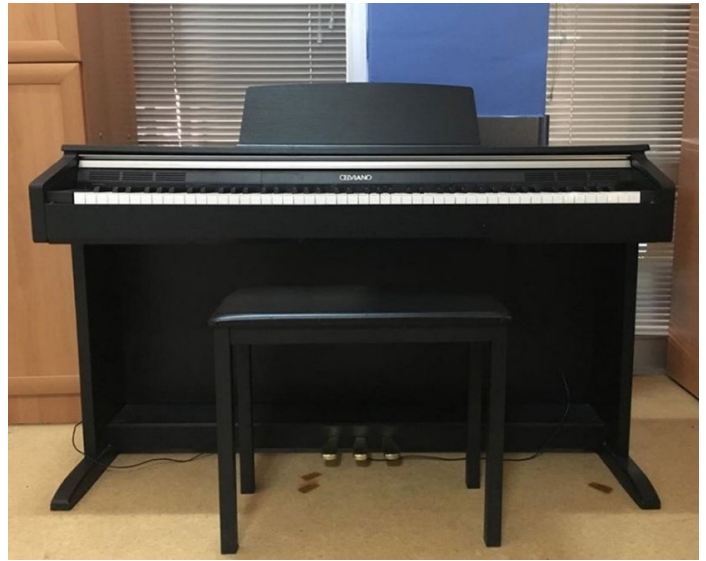

Fig. 3 Digital piano used in training

increase motivation, facilitate understanding of the commands and learning (Fig. 4).

Each session was started with finger warming-up exercises. Patient was allowed to improvise and was told to press the key he wanted as he wanted. After 5 min of warming-up, the fingers were numbered from 1 to 5 , and

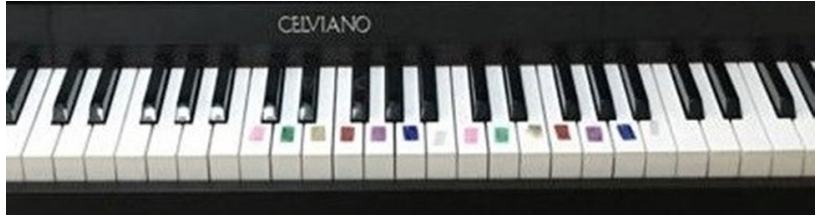

Fig. 4 Coloring of the keys with adhesive papers

the simple 5-scale pentatonic sequence was played with intervals throughout the course.

Following the sequential pentatonic scale training, two hands coordination was trained by pressing the keys of the same color with both hands at the same time.

In the following lessons, a piece was determined according to the music style that the patient liked, and it was aimed to play this piece at the end of 3 months. Also the pieces were taught by symbolizing notes with colors. Training protocol is shown at Table 1 .

\section{Statistical analysis}

SPSS version 25.0 software (IBM, Chicago, IL) was used for statistical data analysis. Descriptive statistics 
Table 1 Neurologic music therapy procedure

\begin{tabular}{|c|c|c|}
\hline & \multicolumn{2}{|l|}{ Procedure } \\
\hline \multirow[t]{3}{*}{1.} & \multicolumn{2}{|l|}{ Preparation } \\
\hline & $\begin{array}{l}\text { The patient sits comfortably on the } \\
\text { chair. }\end{array}$ & \\
\hline & $\begin{array}{l}\text { The forearm and elbow positions are } \\
\text { adjusted. }\end{array}$ & \\
\hline \multirow[t]{2}{*}{2.} & \multicolumn{2}{|l|}{ Improvisation } \\
\hline & $\begin{array}{l}\text { The patient is asked to press the keys } \\
\text { as desired for } 5 \text { minutes. }\end{array}$ & \\
\hline \multirow[t]{4}{*}{3.} & Training & \\
\hline & $\begin{array}{l}\text { The patient is asked to press the keys } \\
\text { from } \mathrm{F} 1 \text { to } \mathrm{F} 5 \text { with the right hand and } \\
\text { then the left hand, respectively. }\end{array}$ & $\frac{\text { MID }}{-\frac{20}{30}}$ \\
\hline & $\begin{array}{l}\text { The patient is asked to press two keys } \\
\text { of the same color at the same time } \\
\text { with the same numbered fingers of } \\
\text { both hands. }\end{array}$ & tryar \\
\hline & $\begin{array}{l}\text { The patient is asked to press a certain } \\
\text { color key with a numbered finger (ex: } \\
\text { press the blue key with } \mathrm{F} 3 \text { of the right } \\
\text { hand). }\end{array}$ & E \\
\hline 4. & Playing a musical piece & \\
\hline & $\begin{array}{l}\text { A simple piece determined according to } \\
\text { the musical taste of the patient is } \\
\text { taught with the coloring of the keys } \\
\text { and finger numbers. }\end{array}$ & \\
\hline
\end{tabular}

are presented together with mean and standard deviation values for continuous variables. Frequency values (number of cases) for categorical variables are shown together with their percentages. For categorical variables, whether there was a significant difference between the groups was determined by Chi-square test when the assumptions were provided, and by the Fisher Exact test when the assumptions were VIOLATED. Whether there is a difference between the two groups for continuous variables was analyzed by non-parametric Mann-Whitney $U$ test. Differences between measurements at two different times were analyzed with the non-parametric Wilcoxon signed ranks test. The relationship between continuous variables was calculated with the Spearman rho correlation coefficient. When evaluating the results of the analysis, $p<0.05$ value was considered statistically significant.

A post hoc power analysis was conducted using G*Power 3 (Faul, Erdfelder, Lang, \& Buchner, 2007) to test the difference between related group means using a two-tailed test, with sample of 9 participants $n=9$ and alpha of 0.05 and to test the difference between two independent group means using a two-tailed test, with a total sample of 18 participants with two equal sized groups of $n=9$ and alpha of 0.05 .

\section{Results}

Descriptive statistics of the participants are shown in Table 2.

A statistically significant improvement was observed in all measurements taken after the training compared to the measurements taken before the training (Table 3).

The key pressing power of fingers for the right and left hand was measured before and after piano training with the Cubase MIDI program. In the patient group $(n=9)$, it was observed that the key pressing powers was statistically

Table 2 Demographic characteristics of the participants

\begin{tabular}{lll}
\hline & CP group $(n=9)$ & Control group $(n=9)$ \\
\hline Gender (F/M) & $5 / 4$ & $4 / 5$ \\
& $55.6 \% / 44.4 \%$ & $44.4 \% / 55.6 \%$ \\
Age (years) & $12.33(\mathrm{SD}: 1.58)$ & 12.44 (SD: 1.67$)$ \\
& $(11-16)$ & $(11-16)$ \\
CP type & & \\
Spastic hemiplegic & $5(55.6 \%)$ & \\
Spastic diplegic & $4(44.4 \%)$ & \\
\hline
\end{tabular}


Table 3 Patient group $(n=9)$ comparison of measurements before and after intervention

\begin{tabular}{|c|c|c|c|c|c|c|c|}
\hline \multirow{2}{*}{ Tests } & \multicolumn{2}{|l|}{ Before } & \multicolumn{2}{|l|}{ After } & \multirow[t]{2}{*}{$Z$} & \multirow[t]{2}{*}{$p$} & \multirow[t]{2}{*}{ Power } \\
\hline & Mean & SD & Mean & SD & & & \\
\hline MACS & 2.89 & 0.93 & 2.44 & 0.52 & $-2.00^{\mathrm{a}}$ & $<0.05$ & 0.79 \\
\hline Right-hand BBT (box/min) & 23.11 & 13.43 & 28.33 & 16.92 & $-2.53^{\mathrm{a}}$ & $<0.05$ & 0.97 \\
\hline Left-hand BBT (box/min) & 28.56 & 13.91 & 32.78 & 16.23 & $-2.53^{\mathrm{a}}$ & $<0.05$ & 0.99 \\
\hline Right hand NHPT (s) & 171.37 & 209.84 & 125.88 & 137.96 & $-2.67^{b}$ & $<0.01$ & 0.37 \\
\hline Left -hand NHPT (s) & 60.04 & 41.09 & 55.42 & 35.63 & $-2.67^{b}$ & $<0.01$ & 0.58 \\
\hline Right-hand dynamometer (lb) & 13.11 & 9.96 & 18.22 & 11.81 & $-2.53^{\mathrm{a}}$ & $<0.05$ & 0.99 \\
\hline Left-hand dynamometer (lb) & 18.33 & 8.66 & 24.11 & 11.82 & $-2.57^{\mathrm{a}}$ & $<0.05$ & 0.99 \\
\hline
\end{tabular}

MACS Manual Ability Classification System, BBT box block test, NHPT nine-hole peg test

Statistically significant at the level of ${ }^{\mathrm{a}} p<0.05,{ }^{\mathrm{b}} p<0.01$ and ${ }^{\mathrm{c}} p<0.001$

\begin{tabular}{|c|c|c|c|c|c|c|c|}
\hline \multirow[t]{2}{*}{ Fingers } & \multicolumn{2}{|l|}{ Before } & \multicolumn{2}{|l|}{ After } & \multirow[t]{2}{*}{$Z$} & \multirow[t]{2}{*}{$p$} & \multirow[t]{2}{*}{ Power } \\
\hline & Mean & SD & Mean & SD & & & \\
\hline Right F1 & 83.89 & 26.31 & 87.67 & 22.24 & $-2.25^{\mathrm{a}}$ & $<0.05$ & 0.66 \\
\hline Right F2 & 89.00 & 20.67 & 92.89 & 18.70 & $-2.11^{\mathrm{a}}$ & $<0.05$ & 0.99 \\
\hline Right F3 & 80.78 & 24.85 & 90.89 & 17.81 & $-2.52^{\mathrm{a}}$ & $<0.05$ & 0.95 \\
\hline Right F4 & 75.11 & 28.89 & 82.56 & 23.47 & $-2.67^{b}$ & $<0.01$ & 0.94 \\
\hline Right F5 & 70.22 & 27.20 & 77.00 & 20.74 & -1.96 & 0.50 & \\
\hline Left F1 & 84.33 & 23.34 & 92.56 & 15.61 & -1.90 & 0.06 & \\
\hline Left F2 & 89.33 & 20.57 & 95.78 & 12.88 & $-1.98^{a}$ & $<0.05$ & 0.57 \\
\hline Left F3 & 87.78 & 14.05 & 90.56 & 12.22 & -1.43 & 0.15 & \\
\hline Left F4 & 83.33 & 13.18 & 89.56 & 11.56 & $-2.67^{b}$ & $<0.01$ & 1.00 \\
\hline Left F5 & 71.11 & 19.83 & 75.78 & 18.64 & $-2.69^{b}$ & $<0.01$ & 1.00 \\
\hline
\end{tabular}

Statistically significant at the level of ${ }^{\mathrm{a}} p<0.05,{ }^{\mathrm{b}} p<0.01$
Table 4 Comparison of MIDI values of the fingers before and after intervention in the patient group $(n=9)$ significantly higher for four of the right-hand fingers before and after piano training (Table 4). For the fifth finger, the $p$ value was found to be 0.50 at the border.

In the patient group $(n=9)$, it was observed that the key pressing powers was statistically significantly higher for three of the fingers in left hand (left 2, left 4, left 5) after piano training (Table 4). For the other two fingers (left $\mathrm{F} 1$, left F3), the difference in the pressing strength of the keys before and after piano training was not found to be statistically significant.

The results of the measurements made in the patient group before and after piano training were compared with the measurements taken from the control group. Except for the left hand grip strength measurements, both the initial values and the final values of the patient group show a statistically significant difference compared to the values of the control group (Table 5).

The key pressing forces of the patient group before and after the training and the control group are shown in Table 6. The finger with the highest strength was $\mathrm{P} 2$ and lowest strength was P5 in both hands before and after the training.
Table 5 Comparison of patient group (before and after intervention) and control group measurements

\begin{tabular}{|c|c|c|c|c|}
\hline & & $Z$ & $p$ & Power \\
\hline \multirow[t]{2}{*}{ Right hand BBT } & $\mathrm{CP}$ before-control & $-3.58^{c}$ & $<0.001$ & 0.99 \\
\hline & $\mathrm{CP}$ after-control & $-3.31^{\mathrm{b}}$ & 0.001 & 0.99 \\
\hline \multirow[t]{2}{*}{ Left-hand BBT } & $\mathrm{CP}$ before-control & $-3.45^{\mathrm{b}}$ & 0.001 & 0.99 \\
\hline & $\mathrm{CP}$ after-control & $-2.66^{\mathrm{b}}$ & 0.008 & 0.91 \\
\hline \multirow[t]{2}{*}{ Right-hand NHPT } & $\mathrm{CP}$ before-control & $-3.10^{\mathrm{b}}$ & 0.002 & 0.49 \\
\hline & $\mathrm{CP}$ after-control & $-2.88^{\mathrm{b}}$ & 0.004 & 0.53 \\
\hline \multirow[t]{2}{*}{ Left-hand NHPT } & $\mathrm{CP}$ before-control & $-2.83^{b}$ & 0.005 & 0.64 \\
\hline & $\mathrm{CP}$ after-control & $-2.70^{\mathrm{b}}$ & 0.007 & 0.64 \\
\hline \multirow{2}{*}{$\begin{array}{l}\text { Right-hand } \\
\text { dynamometer }\end{array}$} & $\mathrm{CP}$ before-control & $-3.11^{\mathrm{b}}$ & 0.002 & 0.93 \\
\hline & $\mathrm{CP}$ after-control & $-2.63^{\mathrm{b}}$ & 0.009 & 0.76 \\
\hline \multirow{2}{*}{$\begin{array}{l}\text { Left-hand } \\
\text { dynamometer }\end{array}$} & $\mathrm{CP}$ before-control & -1.96 & 0.05 & 0.65 \\
\hline & $\mathrm{CP}$ after-control & -1.03 & 0.30 & \\
\hline
\end{tabular}

$B B T$ box block test, $N H P T$ nine-hole peg test

Statistically significant at the level of ${ }^{\mathrm{a}} p<0.05,{ }^{\mathrm{b}} p<0.01$ and ${ }^{\mathrm{c}} p<0.001$ 
Table 6 Patient group (before and after intervention) and control group right-hand fingers MIDI measurements

\begin{tabular}{|c|c|c|c|c|c|c|}
\hline \multirow[t]{3}{*}{ Fingers } & \multicolumn{4}{|c|}{ CP group $(n=9)$} & \multicolumn{2}{|c|}{ Control group $(n=9)$} \\
\hline & \multicolumn{2}{|l|}{ Before } & \multicolumn{2}{|l|}{ After } & \multirow[b]{2}{*}{ Mean } & \multirow[b]{2}{*}{ SS } \\
\hline & Mean & SD & Mean & SD & & \\
\hline Right F1 & 83.89 & 26.31 & 87.67 & 22.24 & 104.56 & 7.54 \\
\hline Right F2 & 89.00 & 20.67 & 92.89 & 18.70 & 109.78 & 6.26 \\
\hline Right F3 & 80.78 & 24.85 & 90.89 & 17.81 & 110.44 & 7.50 \\
\hline Right F4 & 75.11 & 28.89 & 82.56 & 23.47 & 103.22 & 8.14 \\
\hline Right F5 & 70.22 & 27.20 & 77.00 & 20.74 & 101.22 & 9.07 \\
\hline Left F1 & 84.33 & 23.34 & 92.56 & 15.61 & 106.89 & 8.94 \\
\hline Left F2 & 89.33 & 20.57 & 95.78 & 12.88 & 111.56 & 5.59 \\
\hline Left F3 & 87.78 & 14.05 & 90.56 & 12.22 & 109.11 & 8.30 \\
\hline Left F4 & 83.33 & 13.18 & 89.56 & 11.56 & 103.22 & 9.78 \\
\hline Left F5 & 71.11 & 19.83 & 75.78 & 18.64 & 98.67 & 10.68 \\
\hline
\end{tabular}

Table 7 Comparison of the patient group (before and after intervention) and control group right and left hand fingers MIDI measurements

\begin{tabular}{llll}
\hline & & $Z$ & $p$ \\
\hline Right F1 & CP before-control & -1.81 & 0.070 \\
& CP after-control & -1.68 & 0.092 \\
Right F2 & CP before-control & $-2.26^{\mathrm{a}}$ & 0.024 \\
& CP after-control & -1.95 & 0.051 \\
Right F3 & CP before-control & $-2.96^{\mathrm{b}}$ & 0.003 \\
& CP after-control & $-2.61^{\mathrm{b}}$ & 0.009 \\
Right F4 & CP before-control & $-2.53^{\mathrm{a}}$ & 0.012 \\
& CP after-control & $-2.03^{\mathrm{a}}$ & 0.042 \\
Right F5 & CP before-control & $-2.74^{\mathrm{b}}$ & 0.006 \\
& CP after-control & $-2.70^{\mathrm{b}}$ & 0.007 \\
Left F1 & CP before-control & $-2.26^{\mathrm{a}}$ & 0.025 \\
& CP after-control & $-2.03^{\mathrm{a}}$ & 0.042 \\
Left F2 & CP before-control & $-2.88^{\mathrm{b}}$ & 0.004 \\
& CP after-control & $-2.83^{\mathrm{b}}$ & 0.005 \\
Left F3 & CP before-control & $-2.70^{\mathrm{b}}$ & 0.007 \\
& CP after-control & $-2.74^{\mathrm{b}}$ & 0.006 \\
Left F4 & CP before-control & $-2.92^{\mathrm{b}}$ & 0.004 \\
& CP after-control & -1.95 & 0.052 \\
Left F5 & CP before-control & $-3.09^{\mathrm{b}}$ & 0.002 \\
& CP after-control & $-2.78^{\mathrm{b}}$ & 0.005 \\
\hline
\end{tabular}

Statistically significant at the level of ${ }^{\mathrm{a}} p<0.05,{ }^{\mathrm{b}} p<0.01$ and ${ }^{\mathrm{c}} p<0.001$

The differences between patients and control groups in the key pressing power of the right and left hand fingers are shown in Table 7.

The differences between BBT, NHPT and hand dynamometer measurements between the two hands in 5 hemiplegic patients were also evaluated. In all patients, the difference between both hands decreased for BBT and NHPT before and after the training, but the difference between the two hands increased for hand dynamometer even though hand grip strength improved in both hands (Table 8).

\section{Discussion}

In our study, the effect of 3-month Therapeutic Instrumental Music Performance (TIMP) method of Neurologic Music Therapy on upper extremity motor functions in adolescent cerebral palsy patients was investigated.

Muscle strength, functionality and gross and fine motor skills are can be impaired in the upper extremity in cerebral palsy patients $[18,19]$. Also in this study, the CP group was significantly behind in all evaluation parameters compared to the control group.

The literature on the active use of music in upper extremity rehabilitation in cerebral palsy is limited, and studies have generally been conducted on pediatric patients. The literature on active musical instrument education consists mostly of stroke rehabilitation studies today [20-22].

Schneider et al. [23] and Altenmüller et al. [21] investigated the benefits of functional music education in a stroke rehabilitation program involving playing active musical instruments. After 3 weeks of music training, patients showed a significant improvement in their fine and gross motor skills in terms of speed, sensitivity and smoothness of arm movements, while there was almost no difference in the group receiving conventional therapy.

In this study, piano training, which provides an average of five blocks increase in BBT, can be interpreted as advantageous compared to other therapies such as constraintinduced therapy, where gains of 4 blocks were reported after intense arm restriction in stroke patients [24, 25]. Since the piano training intervention is based on commercially available equipment, it also has the potential to be self-directed 
Table 8 Changes in BBT, NHPT and hand dynamometer between two hands in hemiplegic patients before and after intervention

\begin{tabular}{|c|c|c|c|c|c|c|}
\hline & \multicolumn{2}{|c|}{ BBT (box) } & \multicolumn{2}{|c|}{ NHPT (s) } & \multicolumn{2}{|c|}{$\begin{array}{l}\text { Hand dynamometer } \\
\text { (lb) }\end{array}$} \\
\hline & Before & After & Before & After & Before & After \\
\hline Patient 1 & 5 & 7 & 467 & 247 & 14 & 18 \\
\hline Patient 2 & 32 & 28 & 383 & 305 & 28 & 36 \\
\hline Patient 3 & 5 & 7 & 11.17 & 9 & 10 & 15 \\
\hline Patient 4 & 2 & 5 & 44.05 & 30.23 & 5 & 5 \\
\hline Patient 5 & 20 & 16 & 155 & 116 & 20 & 27 \\
\hline
\end{tabular}

and maintaining treatment without limited to the rehabilitation time in a facility.

In this study, significant improvements were observed in key pressing powers of all fingers after piano training. The fingers that benefited the most were 4 th and 5 th fingers of the right hand, and 4th and 5th fingers of the left hand. This finding can be explained by the fact that these fingers also have less pre-training strength than other fingers, children do not prefer to use these fingers in daily life, these fingers started to be used actively in piano training. In literature, 2nd and 3rd fingers are tend to be stronger than 4th and 5th fingers [26, 27]. In addition, the statistical difference in the 4 th and 5 th fingers may be due to the fact that the key pressing movement induced by piano playing is associated with increased corticospinal excitability with both abduction and adduction movements [28]. Overall, these findings show that piano training as a motor skill training method can be an effective finger rehabilitation tool for adolescents with $\mathrm{CP}$.

In the study of Chong et al. [29], 5 adult cerebral palsy patients were given 12 sessions and $30 \mathrm{~min}$ of piano training, and the key pressing speeds were compared. At the end of the training, a statistically insignificant improvement was noted in the key pressing speed of all fingers and it was shown that the difference between the affected hand of patients with $\mathrm{CP}$ and the nondominant hand of healthy adults decreased significantly after training. Also in this study, in the spastic hemiplegic cerebral palsy group before and after training, the difference between the two hands decreased in the box block test and the nine-hole peg test, and although there was a significant improvement in grip strength in both hands, the difference between two hands were increased. With the coordinated use of both hands, it can be said that the positive effect of piano training on gross and fine motor skills is more on the affected side, but the unimpaired/less impaired limb tends to gain more grip strength.

Statistically significant improvement was recorded in all parameters of MACS, box block test, nine-hole peg test and hand grip strength before and after intervention. The statistical difference with the control group before and after the training decreases in most of the parameters. On the other hand, the difference in left hand grip strength between the patient and the control group, which was significant before the training, became statistically insignificant after the training. This finding can be explained by the low left-hand grip strength of all patients in the control group, whose right hand is dominant.

In the study conducted by Lampe et al. [30], a total of 18 children, 10 from $\mathrm{CP}$ and 8 from the other GRMCD (global retardation and movement coordination disorders) group, were enrolled, and the children received 35-40 min of piano training twice a week for 18 months. Significant decrease in the time interval of consecutive key pressing and a significant improvement in the regularity of the time interval for pressing the keys with piano training. There was no difference between CP and other neurodevelopmental diseases, but it was found that the child with poor hand skills showed more progress. In our study, the most improvement was observed in the left hand grip strength, strengths of 4th finger of the right and 4th and 5th fingers of the left hand, which were the weakest at first. In the same study, contrary to this study, there was no significant difference in box block test and hand grip strength. But, since there were no control groups and the fact that the tests were repeated after a long time such as 18 months, the differences found could not be distinguished from ageexpected physical development. It was interpreted that piano training improves functions requiring manual dexterity rather than power. However, in this study, it was seen that both the grip strength and the key pressing powers of the fingers significantly improved with piano training. It may be thought that the patients enrolled in the study of Lampe et al. were not homogeneous, and patients were involved in the study without seeking mental status criteria, and the compliance of the patients to training might be low in those with mental retardation, and this was the reason for failure of the patients on the grip strength measurement and box block test. In addition, the time interval for consecutive keystrokes and the regularity of the key pressing time interval are variables that depend entirely on the sense of rhythm, and even in healthy people with high IQ levels, musical intelligence can be determined to be low because it is a completely different type of intelligence [31]. Therefore, it is not appropriate to use these parameters as variable in studies. 
In the study of Alves-Pinto et al. [10], nine adolescents, seven adult $\mathrm{CP}$ patients and six healthy adolescent control groups were taken and the patients were trained for $8 \mathrm{~h}$ of piano training for four consecutive weeks. Alternatively, a sensorimotor piano system developed specifically for the patients with somatosensory perception deficit and/or spatial perception deficits, applied for ten participants (control + patient) selected by the specialist doctor. In the study, alpha ERD (event-related desynchronization) recording was performed with the task of pressing the keys on the piano, transition speeds between the keys, key pressing forces with the vibration test and reaction time. After 4 weeks of piano training, there was no difference in the keystroke regularity or alpha ERD response of the patients. It was found that piano training was effective in detecting the localization of the vibration on the finger. It was emphasized that longer training would be required to see changes in key press regularity, strength, or alpha ERD. In this study, we also aimed to establish a manual kind of sensorimotor piano system by marking the piano keys with colored adhesive papers and symbolizing patients fingers with numbers. In the study of Alve-Pinto et al., different from this study, we think that the reason for the lack of improvement in the key pressing force and regularity is that the total training time and the sessions are too long to continue to focus. In addition, the results of the study may be controversial because there is no adult control group in the study. It is known that learning ability decreases over time even in healthy adults [32]. Therefore, less impact can be expected in studies on adult patients.

In recently published study of Marrades-Caballero et al. [9], 18 children with severe bilateral CP were divided into 2 groups, and in the first 16 weeks, 9 children received physiotherapy, 9 children received physiotherapy + music therapy, and after 16 weeks, the groups were interchanged. In training, the child was allowed to choose his own instrument and the improvisation method was used, and then a task-specific music education program was applied. In the group receiving music therapy, a significant difference was found in the total score, activity score, arm and hand position, and locomotor scores of the Chailey Levels of Ability scale compared to pre-training, and this significant difference was reported to continue 16 weeks after the treatment. However in the study, severely impaired patients were selected according to both GMFCS (Gross Motor Function Classification System), MACS and CFCS (Communication Function Classification System) and we think that it is a question mark whether it is appropriate to select children with severe communication problems in an education program that requires high level of communication between the teacher and the student, such as instrument training.

In the studies of Chong et al. [20] in stroke patients, box block test and grip strength are strongly correlated with MIDI finger strengths. It has been reported that the strongest correlation between finger powers and box block test has been shown. Also in this study, a strong relationship was found between BBT and NHPT in both hands, and between the individual strengths of all fingers and BBT and NHPT.

In the study of Alves-Pinto et al. [33] where 10 adolescent patients with neuromotor retardation were taken to piano training for 30-45 min 2 days a week for 18 months, patients were evaluated with fMRI during finger opposition, and fMRI changes were evaluated before and after the piano lesson and also with a control group. It was determined that the connectivity between the left primary motor cortex and the right cerebellum increased with piano education. The effects of neurological music therapy on the brain need to be investigated with more studies.

The effects of musical instrument training can extend beyond motor fields and function. We think that neurological music therapy may involve the entire CNS and trigger stronger sensorimotor interactions that lead to coordinated motor responses, unlike other simple motor training.

In the process of continuing neuroplasticity in the development age, methods such as music therapy should be given importance. We think that this method should be included in routine rehabilitation practice, especially in the child and adolescent group. The feasibility of this adaptation should be considered not only in terms of private health institutions, but also in terms of its integration into the national health systems. If the treatment of children with cerebral palsy is not maintained, their integration into society will be interrupted and their dependence on the caregiver will result in loss of workforce, and the economic burden of the person and the caregiver on the state will be greater than the economic burden of this treatment (music therapy and music therapy programs).

The information obtained from the participants' feedback showed that the all participants enjoyed the training program and were motivated in the supervised piano training sessions. All participants completed the 40-min sessions while maintaining a high level of motivation and attention throughout the entire study period. We think that beyond the exercise, targeting and playing a musical piece is an important factor for motivating patients to participate in many repetitive training sessions. We also believe that the "game" nature of this training adds a sense of accomplishment that makes the intervention satisfying.

\section{Limitations}

In our country, hospital follow-up of cerebral palsy patients is disrupted as they get older. The number of patients undergoing follow-up is very low, and among these patients, those who meet the inclusion criteria of the study for neurological music therapy training are much less. The number of 
patients with adolescent cerebral palsy in our country is unknown. For this reason, the power analysis of the study could not be performed before the study, as we were working in a rare population with unknown incidence.

Small sample size and dual role of the researcher as interventionist and evaluator are the limitations of this study. In future studies, it is recommended that the interventionist and the evaluator be separate, and that a third observer be included. Also, one of the limitations of the study is the inability to determine whether the improvement in hand functions continues when the piano education is discontinued, as we cannot repeat the evaluations after a period of piano training due to the COVID-19 pandemic.

\section{Conclusion}

Although the evidence on neurological music therapy is limited, Therapeutic Instrumental Music Performance (TIMP) is a promising method for upper extremity rehabilitation. Neurological music therapy would increase treatment compliance and continuation of adolescent cerebral palsy patients. With TIMP method, which is associated with active music production, improvements and functional gains can be achieved in the grip strength, selective strengths of the fingers, gross and fine hand motor skills of adolescent cerebral palsy patients. It is possible that the patient can plan his/ her own treatment in the future, by communicating with the patient via online video-conferencing, the trainings can be continued remotely, using a program designed specifically for this subject, which transfers key data from the trainer to the patient and from the patient to the trainer. In this way, discontinuations hopefully can be prevented as age advances in adolescent cerebral palsy patients. In order to ensure the applicability of music therapy in our country, competent music therapists should be trained.

Neurological music therapy programs have uncertainties regarding the frequency, duration, and method of application. Although the 3-month, 40-min session duration applied in this study seems to be effective, more studies are needed to establish the piano training protocol in neurological music therapy of patients with cerebral palsy.

\section{Highlights}

- With therapeutic instrumental music performance method, improvements and functional gains can be achieved.

- With this method, treatment interruptions can be avoided, and even the patient can continue a structured program online from home.
- 3-month, 40-min session duration applied in this study seems to be effective protocol, but more studies are needed to establish the optimum training protocol in neurological music therapy of patients with cerebral palsy.

Acknowledgements The authors have stated that they had no interests which might be perceived as posing a conflict or bias. Protocol for this research project has been approved by Ethics Committee of Istanbul Medeniyet University (Decision no: 2018/0199) and the study is registered in clinicaltrials.gov (NCT03778398).

Author contributions All authors have contributed to this research significantly and all authors are in agreement with the content of the manuscript. Material preparation, data collection, analysis were performed by BDK and AI. The first draft was written by BDK and both authors read, commented and approved the final manuscript.

\section{Compliance with ethical standards}

Conflict of interest The author(s) declare that they have no competing interests.

Ethical approval All procedures performed in studies involving human participants were in accordance with the ethical standards of the institutional and/or national research committee and with the 1964 Helsinki declaration and its later amendments or comparable ethical standards. The protocol number is: 2018/0199 from Istanbul Medeniyet University Goztepe Training and Research Hospital Ethics Committee.

Informed consent Informed consent was obtained from all individual participants included in the study.

\section{References}

1. Rosenbloom L (2007) Definition and classification of cerebral palsy. Definition, classification, and the clinician. Dev Med Child Neurol Suppl 109:43

2. Chin TY, Duncan JA, Johnstone BR et al (2005) Management of the upper limb in cerebral palsy. J Pediatr Orthop B 14(6):389-404

3. Bonnier B, Eliasson AC, Krumlinde-Sundholm L (2006) Effects of constraint-1nduced movement therapy in adolescents with hemiplegic cerebral palsy: a day camp model. Scand J Occup Ther 13(1):13-22

4. Levitt S, Addison A (2019) Treatment of cerebral palsy and motor delay, 6th edn. Wiley-Blackwell, Hoboken

5. Ghai S, Ghai I, Effenberg AO (2018) Effect of rhythmic auditory cueing on gait in cerebral palsy: a systematic review and metaanalysis. Neuropsychiatr Dis Treat. 14:43-59

6. Alves-Pinto A, Turova V, Blumenstein T et al (2016) The case for musical instrument training in cerebral palsy for neurorehabilitation. Neural Plast 2016:1072301-1072301

7. Kantor J, Kantorová L, Marečková J et al (2019) Potential of vibroacoustic therapy in persons with cerebral palsy: an advanced narrative review. Int J Environ Res Public Health 16(20):3940

8. Thaut MH, Mcintosh GC, Hoemberg V (2014) Neurologic music therapy: from social science to neuroscience. In: Thaut $\mathrm{MH}$, Hoemberg V (eds) Handbook of neurologic music therapy. OUP, Oxford, pp 1-6

9. Marrades-Caballero E, Santonja-Medina CS, Sanz-Mengibar JM et al (2018) Neurologic music therapy in upper-limb rehabilitation 
1n children with severe bilateral cerebral palsy: a randomized controlled trial. Eur J Phys Rehabil Med 54(6):866-872

10. Alves-Pinto A, Ehrlich S, Cheng G et al (2017) Effects of shortterm piano training on measures of finger tapping, somatosensory perception and motor-related brain activity in patients with cerebral palsy. Neuropsychiatr Dis Treat 13:2705-2718

11. Eliasson AC, Krumlinde-Sundholm L, Rosblad B et al (2006) The manual ability classification system (Macs) for children with cerebral palsy: scale development and evidence of validity and reliability. Dev Med Child Neurol. 48(7):549-554

12. Akpinar P, Tezel CG, Eliasson AC et al (2010) Reliability and cross-cultural validation of the Turkish version of manual ability classification system (Macs) for children with cerebral palsy. Disabil Rehabil. 32(23):1910-1916

13. Araneda R, Ebner-Karestinos D, Paradis J et al (2019) Reliability and responsiveness of the Jebsen-taylor test of hand function and the box and block test for children with cerebral palsy. Dev Med Child Neurol 61(10):1182-1188

14. Poole JL, Burtner PA, Torres TA et al (2005) Measuring dexterity in children using the Nine-Hole Peg Test. J Hand Ther 18(3):348-351

15. Klingels K, De Cock P, Molenaers G et al (2010) Upper limb motor and sensory impairments in children with hemiplegic cerebral palsy. Can they be measured reliably? Disabil Rehabil. 32(5):409-416

16. Mathiowetz V, Wiemer DM, Federman SM (1986) Grip and pinch strength: norms for 6- to 19-year-olds. Am J Occup Ther 40(10):705-711

17. Mathiowetz V, Weber K, Volland G et al (1984) Reliability and validity of grip and pinch strength evaluations. J Hand Surg 9(2):222-226

18. Makki D, Duodu J, Nixon M (2014) Prevalence and pattern of upper limb involvement in cerebral palsy. J Child Orthop 8(3):215-219

19. Manske PR (1990) Cerebral palsy of the upper extremity. Hand Clin 6(4):697-709

20. Chong HJ, Han SJ, Kim Y-J et al (2014) Relationship between output from midi-keyboard playing and hand function assessments on affected hand after stroke. NeuroRehabilitation 35(4):673-80

21. Altenmuller E, Marco-Pallares J, Munte TF et al (2009) Neural reorganization underlies improvement in stroke-1nduced motor dysfunction by music-supported therapy. Ann N Y Acad Sci 1169:395-405

22. Villeneuve M, Penhune V, Lamontagne A (2014) A piano training program to improve manual dexterity and upper extremity function in chronic stroke survivors. Front Hum Neurosci 8:662
23. Schneider S, Schonle PW, Altenmuller E et al (2007) Using musical instruments to improve motor skill recovery following a stroke. J Neurol 254(10):1339-1346

24. Leung DP, Ng AK, Fong KN (2009) Effect of small group treatment of the modified constraint induced movement therapy for clients with chronic stroke in a community setting. Hum Mov Sci 28(6):798-808

25. Siebers A, Oberg U, Skargren E (2010) The effect of modified constraint-1nduced movement therapy on spasticity and motor function of the affected arm in patients with chronic stroke. Physiother Can 62(4):388-396

26. Dickson RA, Calnan JS (1972) Hand function - a practical method of assessment. Br J Surg 59(4):316-317

27. $\mathrm{Bj} \mathrm{M}, \mathrm{Tj} \mathrm{A}, \mathrm{Ja} F$ et al (1996) Keyboard reaction force and finger flexor electromyograms during computer keyboard work. Hum Factors 38(4):654-664

28. Carroll TJ, Riek S, Carson RG (2002) The sites of neural adaptation induced by resistance training in humans. J Physiol 544(Pt 2):641-652

29. Chong HJ, Cho SR, Jeong E et al (2013) Finger exercise with keyboard playing in adults with cerebral palsy: a preliminary study. J Exerc Rehabil 9(4):420-425

30. Lampe R, Thienel A, Mitternacht J et al (2015) Piano training in youths with hand motor impairments after damage to the developing brain. Neuropsychiatr Dis Treat 11:1929-1938

31. Tierney A, White-Schwoch T, Maclean J et al (2017) Individual differences in rhythm skills: links with neural consistency and linguistic ability. J Cogn Neurosci 29(5):855-868

32. Janacsek K, Fiser J, Nemeth D (2012) The best time to acquire new skills: age-related differences in implicit sequence learning across the human lifespan. Dev Sci 15(4):496-505

33. Alves-Pinto A, Turova V, Blumenstein T et al (2015) FMRI assessment of neuroplasticity in youths with neurodevelopmentalassociated motor disorders after piano training. Eur J Paediatr Neurol 19(1):15-28

Publisher's Note Springer Nature remains neutral with regard to jurisdictional claims in published maps and institutional affiliations. 